\title{
openheart Cost-effectiveness of an insertable cardiac monitor in a high-risk population in the UK
}

\author{
Claudia I Rinciog, ${ }^{\circ 1}$ Laura M Sawyer, ${ }^{1}$ Alexander Diamantopoulos, ${ }^{1}$ \\ Mitchell S V Elkind, ${ }^{2}$ Matthew Reynolds, ${ }^{3}$ Stylianos I Tsintzos, ${ }^{4}$ Paul D Ziegler, ${ }^{5}$ \\ Maria E Quiroz, ${ }^{5}$ Claudia Wolff, ${ }^{4}$ Klaus K Witte ${ }^{6}$
}

\begin{abstract}
- Additional material is published online only. To view please visit the journal online (http://dx.doi.org/10. 1136openhrt-2019-001037).
\end{abstract}

To cite: Rinciog $\mathrm{Cl}$, Sawyer LM, Diamantopoulos A, et al. Costeffectiveness of an insertable cardiac monitor in a high-risk population in the UK. Open Heart 2019;6:e001037. doi:10.1136/ openhrt-2019-001037

Received 27 February 2019 Revised 16 April 2019 Accepted 26 April 2019

\section{Check for updates}

(c) Author(s) (or their employer(s)) 2019. Re-use permitted under CC BY-NC. No commercial re-use. See rights and permissions. Published by BMJ.

${ }^{1}$ Symmetron Limited, London, UK

${ }^{2}$ Neurology Department, Columbia University College of Physicians and Surgeons, New York, New York, USA

${ }^{3}$ Cardiovascular Medicine, Baim Institute for Clinical Research, Boston, Massachusetts, USA

${ }^{4}$ Health Economics and Reimbursement, Medtronic International Trading Sarl, Tolochenaz, Switzerland ${ }^{5}$ Cardiac Rhythm and Heart Failure, Medtronic, Mounds View, Minnesota, USA

${ }^{6}$ Division of Cardiovascular and Diabetes Research, University of Leeds, Leeds, UK

Correspondence to Claudia I Rinciog; crinciog@ symmetron.net

\section{ABSTRACT}

Objective To evaluate the cost-effectiveness of insertable cardiac monitors (ICMs) compared with standard of care (SoC) for detecting atrial fibrillation (AF) in patients at high risk of stroke ( $\mathrm{CHADS}_{2}>2$ ), using a UK National Health Service (NHS) perspective.

Methods Using patient characteristics and clinical data from the REVEAL AF trial, a Markov model assessed the cost-effectiveness of detecting AF with an ICM compared with $\mathrm{SoC}$. Costs and benefits were extrapolated across modelled patient lifetime. Ischaemic and haemorrhagic strokes, intracranial and extracranial haemorrhages and minor bleeds were modelled. Diagnostic and device costs were included, plus costs of treating stroke and bleeding events and costs of oral anticoagulants (OACs). Costs and health outcomes, measured as quality-adjusted life years (QALYS), were discounted at 3.5\% per annum. One-way deterministic and probabilistic sensitivity analyses (PSA) were undertaken.

Results The total per-patient cost for ICM was $£ 13360$ versus $£ 11936$ for SoC (namely, annual 24 hours Holter monitoring). ICMs generated a total of $6.50 \mathrm{QALY}$ s versus 6.30 for SoC. The incremental cost-effectiveness ratio (ICER) was $£ 7140 /$ QALY gained, below the $£ 20$ 000/QALY acceptability threshold. ICMs were cost-effective in $77.4 \%$ of PSA simulations. The number of ICMs needed to prevent one stroke was 21 and to cause a major bleed was 37 . ICERs were sensitive to assumed proportions of patients initiating or discontinuing OAC after AF diagnosis, type of OAC used and how intense the traditional monitoring was assumed to be under SoC.

Conclusions The use of ICMs to identify AF in a high-risk population is cost-effective for the UK NHS.

\section{INTRODUCTION}

Atrial fibrillation (AF) has long been associated with stroke risk. ${ }^{12}$ Although anticoagulation therapy can effectively reduce stroke risk in patients with $\mathrm{AF}$, initial diagnosis can be challenging. Symptoms may be non-specific, AF may be intermittent ${ }^{3}$ and current opportunistic short-term monitoring solutions (such as wearing a Holter monitor for 24 hours) are not well suited to detect all AF episodes. Further, correlation of even specific

\section{Key questions}

What is already known about this subject?

- Atrial fibrillation (AF) is a major risk factor for strokes that can be prevented via oral anticoagulation (OAC). Opportunistic, symptom-based, and single time-point diagnostic tests have limited yields. Continuous monitoring with an insertable cardiac monitor (ICM) in the REVEAL AF study demonstrated a high incidence of $A F$ in high-risk patients where previous external monitoring ( $\geq 24$ hours) was negative. The cost-effectiveness of ICM monitoring in this population is unknown.

What does this study add?

- In a population with mean CHADS, score of 2.94 , the cost per quality-adjusted life-year gained with ICM compared with Standard of Care (SoC) was $£ 7140$, a value well below conventional thresholds for cost-effectiveness. Results were sensitive to the cost of SoC and to rates of uptake and duration of $O A C$ therapy after diagnosis.

How might this impact on clinical practice?

- The use of ICMs to detect AF and identify high-risk candidates for OAC improves health outcomes relative to $\mathrm{SoC}$ at an acceptable extra cost to the UK National Health Service.

symptoms and a pathological underlying rhythm can be difficult. ${ }^{4}$

Insertable cardiac monitors (ICMs) can continuously monitor patients for AF. The REVEAL AF study ${ }^{5}$ used Reveal ${ }^{\circledR}$ (Medtronic, Dublin, Ireland) ICM devices to detect AF. The study patients had non-specific symptoms, sufficient stroke risk factors to require oral anticoagulation (OAC) therapy should $\mathrm{AF}$ be detected and no AF found following at least 24 hours of external monitoring performed within 90 days prior to study enrolment. After 18 months, the AF detection rate for episodes lasting at least $6 \mathrm{~min}$ was found to be $29.3 \%$; at 30 months, the rate was $39.8 \%$. 
ICM use in this patient population is clinically effective in diagnosing previously undetected AF but its cost-effectiveness compared with standard care (SoC) in this application is unknown.

\section{METHODS}

A Markov model was created to compare costs and benefits of Reveal ${ }^{\circledR}$ ICM versus SoC in patients with high risk of stroke but no confirmed AF diagnosis. Based on a similar analysis in cryptogenic stroke, ${ }^{6}$ the model estimated monitoring and treatment costs from a UK NHS perspective, and health benefits as quality-adjusted life years (QALYs), discounted at $3.5 \%$ annually. ${ }^{7}$ The model allowed estimation of life-years gained and stroke events avoided. The model cohort was set up to have the same baseline characteristics as recorded in the REVEAL AF clinical trial ${ }^{5}$ (Clinicaltrials.gov, NCT01727297) (see online supplementary file 1 ).

A lifetime horizon captured all relevant costs and health-related quality of life (QoL) effects. Cycle length for recording outcomes was 3 months, matching follow-up intervals in the REVEAL AF trial.

\section{Model structure}

The model estimates both the efficacy and costs of the ICM device itself as well as the potential costs and health effects of timely and accurate diagnosis.

In the model's hypothetical cohort, all patients with underlying $\mathrm{AF}$ are assumed to be undiagnosed at baseline. In each cycle, patients with AF may remain undiagnosed, have existing AF detected, or, for patients with no underlying AF initially, develop AF. Patients in whom $\mathrm{AF}$ is detected are assumed to receive new OAC (NOAC) therapy (non-vitamin-K OAC); by contrast, patients with undetected AF will have no change in their treatment. Thus, patients with undetected AF are assumed not to receive anticoagulation for stroke prevention. Patients who have a stroke will subsequently move to a post-stroke health state, where they are assumed to remain. AF detection status and treatment received can still change in these states, but patients cannot transit to other states except death.

Movement of patients through the model is shown in figure 1.

\section{Input parameters \\ Population}

The patient cohort reflects the baseline characteristics of the REVEAL AF trial population, with $72.6 \%$ receiving aspirin treatment and $20.3 \%$ having a history of stroke events in the year prior to recruitment. The average patient entered the model aged 71.3 and with a virtual $\mathrm{CHADS}_{2}$ score of 2.94. The distribution of $\mathrm{CHADS}_{2}$ stroke risk scores also matched the REVEAL AF trial population (see online supplementary file 2).

\section{Probabilities}

Event probabilities used in the model are shown in table 1 . Values were derived from published studies of baseline risks and calculated via drug treatment effects applied on those risks (see online supplementary file 3).

\section{AF incidence, detection and subsequent treatment}

The model definition of AF corresponded to that used in the REVEAL AF trial. ${ }^{5}$ Base-case AF episodes were defined as lasting for $\geq 6 \mathrm{~min}$, while a scenario analysis explored the impact of AF episodes lasting for $\geq 5.5$ hours (see online supplementary file 2). AF detection rates for patients receiving an ICM (Reveal ${ }^{\circledR}$, Medtronic) were modelled based on the first 30 months of observations from the REVEAL AF trial (figure 2). Beyond month 30, a constant long-term AF risk of $3.9 \%$ per cycle was derived based on average detection rates across the REVEAL AF study (excluding the first 3 months). Because ICMs may fail to identify some episodes of AF, the calculation of $\mathrm{AF}$ incidence incorporated an assumed device sensitivity of $96.1 \%{ }^{8}$

The analysis assumed that on AF detection, all patients were administered NOAC therapy, in accordance with National Institute for Health and Care Excellence (NICE) guidance. ${ }^{9}$ In a scenario analysis, a lower NOAC uptake $(66.35 \%)$ was assumed, based on an average of NOAC uptake from published sources. ${ }^{510}$

\section{Ischaemic stroke risk and severity}

In each cycle, the risk of having an ischaemic stroke (IS) was based on patients' AF status, virtual $\mathrm{CHADS}_{2}$ score, anticoagulation treatment and age (see table 2). IS risk calculation involved synthesis from several publications. ${ }^{111-13}$ IS severity was assigned to a distribution based on published cost-effectiveness analyses ${ }^{14}{ }^{15}$; this distribution was independent of anticoagulation treatment status (see online supplementary file 4 ). The probabilities for events and severity are summarised in table 2.

\section{Bleeding events and anticoagulation therapy changes}

Bleed risks were assumed to be related to NOAC treatment and age. The type and likelihood of bleeds were based on published analyses, ${ }^{14}{ }^{15}$ and their severity (see online supplementary file 4) was independent of any anticoagulation treatment given (see table 1). ${ }^{6}$ Non-fatal extracranial bleeds were assumed to cause temporary (6 week) discontinuation of NOACs in $75 \%$ of patients receiving NOAC and permanent discontinuation in 25\% of patients receiving NOACs. Among patients on aspirin, $25 \%$ were precluded from receiving NOAC in future if they experienced a bleed. ${ }^{16} 17$ Non-fatal intracranial bleeds caused temporary (6 week) discontinuation of NOAC therapy in $44 \%$ of patients on NOAC, whereas $56 \%$ had their NOAC therapy discontinued permanently (or banned from use in future, where the event occurred while the patient was on aspirin). ${ }^{16}{ }^{17}$ Clinically relevant non-major (CRNM) bleed did not lead to discontinuation of NOAC. Haemorrhagic strokes while on NOAC therapy led to discontinuation and switch to aspirin in $100 \%$ of cases-or preclusion from receiving NOAC in future if the patient was on aspirin. NOACs were not restarted in 
PATIENT AF STATUS AND TREATMENT

Patient status on AF diagnosis and treatment is tracked throughout the model in all health states

\section{Cerebrovascular AND BLEEDING EVENTS}

These have either temporary or permanent health consequences

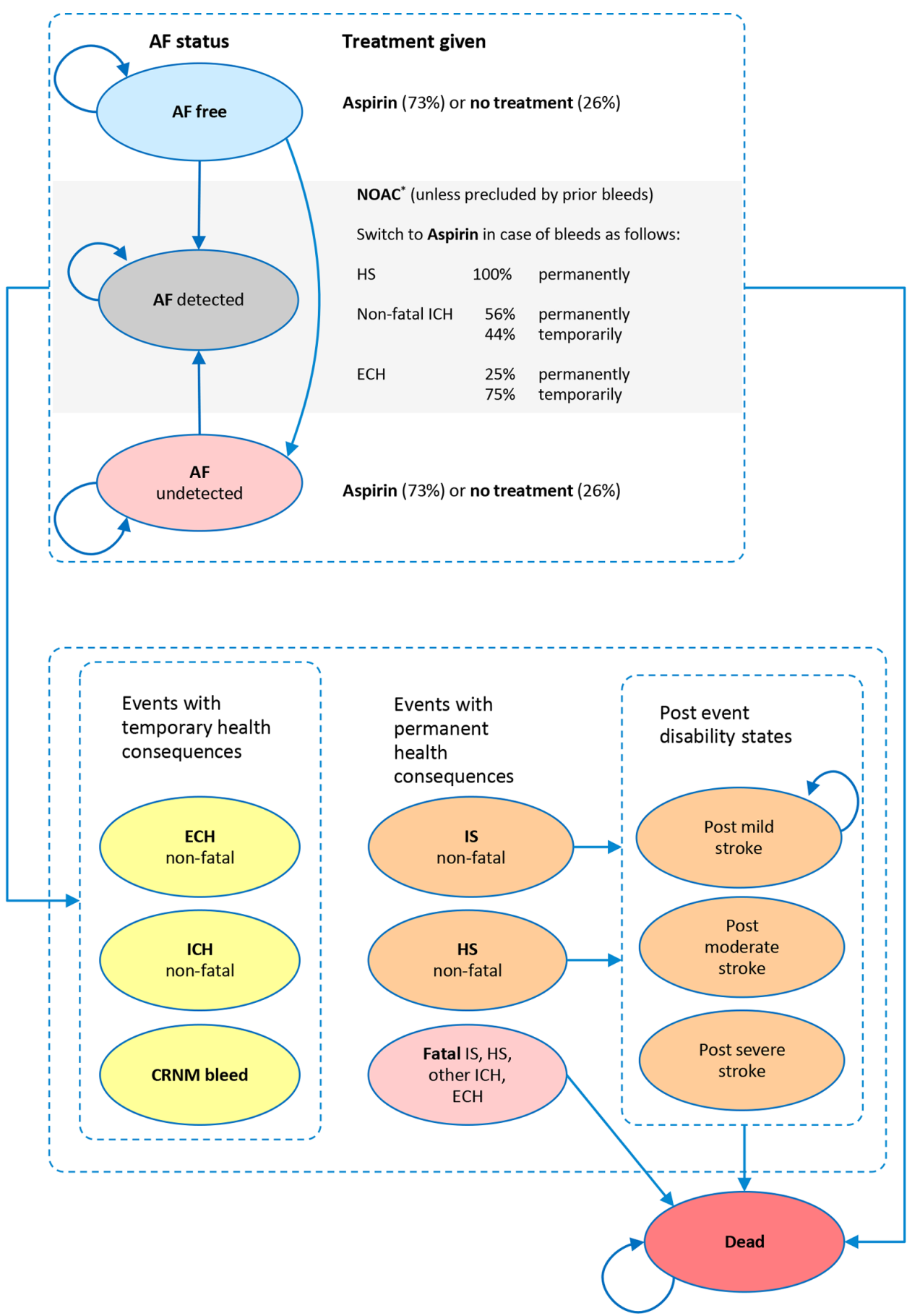

Figure 1 Model flow. *NOACs are administered in base-case analysis and warfarin is substituted in sensitivity analysis. AF, atrial fibrillation; CRNM, clinically relevant non-major; ECH, extracranial haemorrhage; HS, haemorrhagic stroke; ICH, intracranial haemorrhage; IS, ischaemic stroke; NOAC, new oral anticoagulants.

patients with haemorrhagic stroke (HS) even if they later had a positive AF diagnosis. Finally, $16 \%$ of patients on NOAC were assumed to discontinue treatment each year for reasons unrelated to bleeding events. ${ }^{15}$

In terms of cost and QoL impact, non-fatal extracranial haemorrhage, other intracranial haemorrhage (ie, non-haemorrhagic strokes) and CRNM bleeds had temporary consequences applied to the cycle in which the bleed occurred. By contrast, IS and HS were assumed to have permanent consequences for ongoing costs,
QoL and long-term mortality risk, with different levels of impact depending on stroke severity (table 1 ).

\section{Mortality}

Age-dependent background mortality in the model was based on UK life tables adjusted as required to exclude deaths due to cerebrovascular events. ${ }^{18}$ Following non-fatal strokes, mortality risk increased depending on stroke severity and treatment (see online supplementary file 5). 
Table 1 Annual risks and severity of bleeds and haemorrhagic stroke, by anticoagulant treatment received

\begin{tabular}{|c|c|c|c|c|}
\hline & No treatment & Aspirin & NOAC*† & Warfarin* \\
\hline $\mathrm{ICH} \ddagger$ & $0.2 \%$ & $0.5 \%$ & $0.3 \%$ & $0.8 \%$ \\
\hline HS§ & $0.1 \%$ & $0.3 \%$ & $0.2 \%$ & $0.5 \%$ \\
\hline Other ICH & $0.1 \%$ & $0.2 \%$ & $0.1 \%$ & $0.3 \%$ \\
\hline $\mathrm{ECHף}$ & $2.0 \%$ & $2.5 \%$ & $2.7 \%$ & $3.1 \%$ \\
\hline Gl bleed & $0.8 \%$ & $1.0 \%$ & $1.1 \%$ & $1.3 \%$ \\
\hline Other ECH & $1.2 \%$ & $1.4 \%$ & $1.6 \%$ & $1.8 \%$ \\
\hline All major bleeds & $2.2 \%$ & $2.9 \%$ & $3.1 \%$ & $3.8 \%$ \\
\hline CRNM bleed & $5.3 \%$ & $6.9 \%$ & $7.9 \%$ & $9.5 \%$ \\
\hline HS severity & \multicolumn{4}{|c|}{ Mild: $28 \%$; moderate: $23 \%$; severe: $12 \%$ fatal: $37 \%{ }^{1415}$} \\
\hline Case fatalities & \multicolumn{4}{|c|}{ Due to other ICH: $13 \%$, due to $\mathrm{ECH}: 2 \%{ }^{1415}$} \\
\hline
\end{tabular}

*NOAC was used as treatment in base-case analysis and warfarin was considered in sensitivity analysis.

†A class effect was assumed by taking the average across apixaban, dabigatran (low and high dose), rivaroxaban, edoxaban (low and high dose). ${ }^{11}$

$\neq \mathrm{ICHs}(59.7 \%)$ were assumed to be $\mathrm{HS} .^{1415}$

$\S \mathrm{HS}$ risk was adjusted by a factor of 1.97 (95\% Cl 1.79 to 2.16$)$ per decade. ${ }^{31}$

|ECHs $(41.8 \%)$ were assumed to be Gl bleeds. ${ }^{14} 15$

CRNM, clinically relevant non-major; ECH, extracranial haemorrhage; Gl, gastrointestinal; HS, haemorrhagic stroke; ICH, extracranial

haemorrhage; NOAC, new oral anticoagulant.

Health-related QOL

Baseline EuroQol-five dimension data came from the REVEAL AF study. Utility data for health states and acute events were adapted from a similar analysis in patients with cryptogenic stroke, ${ }^{6}$ adjusting for the absence of stroke history in majority of patients. ${ }^{14} 151920$ Modelled utilities are presented in table 3 and further detailed in the online supplementary file 6 .

\section{Resource use and costs \\ ICM device costs}

The model accounted for one-time costs associated with inserting the ICM and subsequently removing it after battery expiry (assumed 3 years after insertion). Per cycle, costs were applied for ICM patients to cover visiting a healthcare professional if $\mathrm{AF}$ was detected, as well as regular ICM diagnostics, interrogation and unscheduled device-related visits. Unscheduled visit frequency was based on rates in the REVEAL AF trial. Patients with undetected AF continued SoC after their ICM was

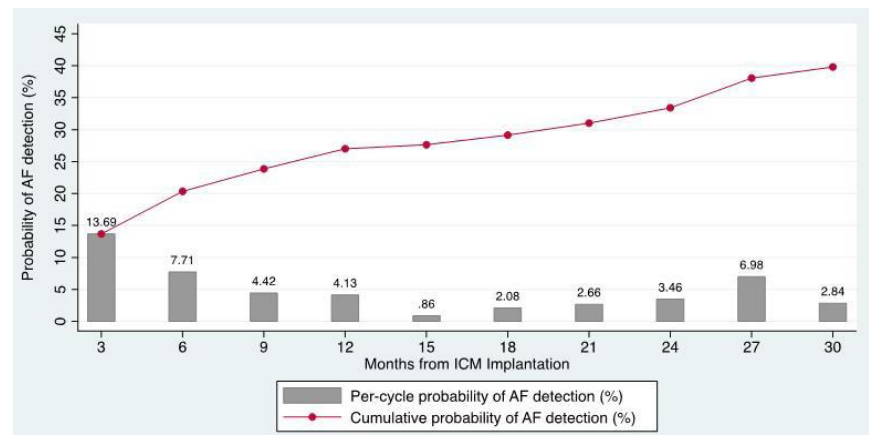

Figure 2 AF detection rates in REVEAL AF-all patients. AF, atrial fibrillation; ICM, insertable cardiac monitor. removed. There was also a risk $(2.9 \%)$ of unplanned ICM removal before 3 years due to a range of clinical, technical or personal factors (Medtronic data on file).

\section{Other costs}

OAC treatment costs were estimated as an average of all European Medicines Agency (EMA) licensed NOACs: apixaban, dabigatran, edoxaban and rivaroxaban (see online supplementary file 7 ).

Acute events, such as strokes and bleeds, were associated with high short-term costs. When they resulted in permanent health consequences, ongoing costs were attached to being in a 'post-stroke' health state. Health state and event costs are summarised in table 3 .

SoC costs and diagnostic yield assumptions

The REVEAL AF study did not contain information about SoC monitoring costs and diagnostic yield. We assumed that patients whose AF risk matched the REVEAL AF trial could be reasonably expected to receive a 24 hours Holter monitor once per year. Holter monitor costs were assumed to be captured by the UK NHS reference cost for ECG monitoring and stress testing. ${ }^{21}$

Relative diagnostic yield of ICM versus SoC was defined by an estimated HR of 33.9 (95\% CI 13.2 to NE), based on a comparison of $\mathrm{AF}$ monitoring strategies in patients at high risk of $\mathrm{AF}$ and stroke, using REVEAL AF clinical trial data. ${ }^{22}$ After ICM explantation, we assumed that patients would be followed up and detected with AF according to SoC-specific risks.

\section{Analyses}

An incremental cost-effectiveness ratio (ICER) was calculated for ICM versus SoC, considering QALYs and 
Table 2 Annual stroke risk and severity by $\mathrm{CHADS}_{2}$ risk score, AF status and anticoagulant treatment received

\begin{tabular}{|c|c|c|c|c|c|c|}
\hline \multirow[b]{2}{*}{ CHADS $_{2}$ score } & \multicolumn{2}{|l|}{ No AF } & \multicolumn{4}{|l|}{ AF } \\
\hline & No treatment & Aspirin & No treatment & Aspirin & NOAC*T $^{*}$ & Warfarin* \\
\hline 0 & $0.2 \%$ & $0.2 \%$ & $1.1 \%$ & $0.8 \%$ & $0.3 \%$ & $0.3 \%$ \\
\hline 2 & $1.3 \%$ & $0.9 \%$ & $6.3 \%$ & $4.5 \%$ & $1.7 \%$ & $1.7 \%$ \\
\hline 3 & $2.5 \%$ & $1.8 \%$ & $12.0 \%$ & $8.6 \%$ & $3.3 \%$ & $3.2 \%$ \\
\hline 5 & $3.6 \%$ & $2.6 \%$ & $17.2 \%$ & $12.3 \%$ & $4.7 \%$ & $4.6 \%$ \\
\hline 6 & $4.0 \%$ & $2.9 \%$ & $19.2 \%$ & $13.7 \%$ & $5.3 \%$ & $5.1 \%$ \\
\hline IS severity & \multicolumn{6}{|c|}{ Mild: 42\%; moderate: $26 \%$; severe: $10 \%$; fatal: $22 \%$} \\
\hline
\end{tabular}

*NOAC was used as treatment in base-case analysis and warfarin was considered in sensitivity analysis.

†A class-effect for NOAC was assumed by taking the average efficacy of apixaban, dabigatran (low and high dose), rivaroxaban, edoxaban (low and high dose). ${ }^{11}$

fIS risk was adjusted by a factor of 1.46 (95\% Cl 0.8 to 2.16$)$ per decade. ${ }^{13}$

$\mathrm{AF}$, atrial fibrillation; IS, ischaemic stroke; NOAC, new oral anticoagulant.

healthcare costs accumulated over the cohort lifetime. Willingness-to-pay thresholds of $£ 20000$ and $£ 30000$ per QALY gained were considered. ${ }^{7}$ Deterministic one-way sensitivity analyses were performed on all model parameters including baseline characteristics, diagnostic accuracy, treatment efficacy and safety, utility and cost values. A scenario where warfarin was given instead of NOACs was analysed, and treatment discontinuation probabilities were studied in separate analyses. A probabilistic sensitivity analysis was performed (1000 samples) with appropriate distributions fitted to model values.

\section{RESULTS}

\section{Base-case analysis}

The base-case deterministic analysis found that ICM provided a benefit over SoC of 0.1994 QALYs at an incremental cost of £1424 across patient lifetime and led to 4.8 fewer strokes per 100 patients (table 4 ). The base-case ICER for ICM versus SoC was $£ 7140$ per QALY gained, suggesting ICM is a cost-effective intervention for use in the UK NHS. The number of ICMs needed to prevent one stroke was 21 and to cause a major bleed was 37 .

Incremental benefits and costs were estimated for 3 years of ICM monitoring. ICM monitoring had higher initial costs compared with SoC; it was also associated with slightly higher health state and bleed-related costs. However, by reducing the rates of IS events, use of ICMs generated cost-savings both from IS event costs as well as post-stroke health state costs (total stroke-related costs for ICM were $£ 3783$ versus $£ 4270$ for SoC).

The probabilistic sensitivity analysis showed that differences in the costs and benefits between strategies were statistically significant (table 4 ). The ICM strategy had a $77.4 \%$ probability of being more cost-effective at a threshold of $£ 20000$ per QALY gained; that figure rose to $86.4 \%$ at a threshold of $£ 30000$. The associated cost-effectiveness acceptability curve is presented in the online supplementary file 8 .

\section{Subgroup analysis}

The base-case analysis modelled a distribution of $\mathrm{CHADS}_{2}$ scores matching the REVEAL AF study population, with an average value of $2.94 .^{5}$

The table 5 shows total costs, QALYs and ICER for SoC and ICM calculated separately by CHADS $_{2}$ score subgroups. When the analysis was carried out only in patients with a CHADS 2 score of 2, the ICER for ICM versus $\mathrm{SoC}$ was higher compared with the base-case analysis. In patients with a CHADS $_{2}$ score of 3 , the ICER was closer to the base-case analysis because $\mathrm{CHADS}_{2}=3$ was similar to the average in the base-case analysis. In patients with higher risk CHADS $_{2}$ scores (range 4-6), the ICER was higher than in the base-case. This increase can be attributed to the lower $\mathrm{AF}$ detection rates observed in the REVEAL AF trial for the $\mathrm{CHADS}_{2}=3-4$ subgroup compared with the all-patients population (base-case).

\section{Scenario analysis}

The table 5 also shows results for several scenarios that were deemed clinically relevant, as they addressed essential model assumptions and data inputs. When the assumed NOAC given after AF detection was substituted with warfarin, the ICER increased slightly. This was driven by an increase in costs and a decrease in total QALYs in the ICM group, both caused by the higher bleed risks of warfarin. The higher costs associated with adverse events on warfarin eclipsed any potential cost-saving brought by the lower drug acquisition cost ( $£ 5.71$ per cycle for warfarin vs $£ 159.43$ for NOAC). In the scenario where OAC treatment discontinuation was only allowed in cases of bleeding (ie, patients were not allowed to discontinue for other reasons), the ICER improved, in large part due to the continued protection afforded against IS for a greater proportion of the population. Other parameters 
Table 3 Cost and utilities of interventions, events and health states

\begin{tabular}{|c|c|c|c|c|c|}
\hline Event, intervention or health state & $\begin{array}{l}\text { Mean cost } \\
(£)\end{array}$ & SE (£) & Mean utility & SE & Source \\
\hline \multicolumn{6}{|l|}{ Stroke and bleed events* } \\
\hline Mild IS & 3783 & 997 & 0.730 & 0.014 & \multirow{8}{*}{$\begin{array}{l}\text { Luengo-Fernandez et al } \\
(2013 \mathrm{a}, \mathrm{b}){ }^{1920} \dagger\end{array}$} \\
\hline Moderate IS & 19737 & 2451 & 0.500 & 0.037 & \\
\hline Severe IS & 26957 & 3947 & 0.130 & 0.057 & \\
\hline Fatal IS & 3403 & 551 & 0.000 & - & \\
\hline Mild HS & 11016 & 2017 & 0.730 & 0.014 & \\
\hline Moderate HS & 28301 & 5563 & 0.500 & 0.037 & \\
\hline Severe HS & 47872 & 12911 & 0.130 & 0.057 & \\
\hline Fatal HS & 1771 & 667 & 0.000 & - & \\
\hline $\begin{array}{l}\text { Disutility for all recurrent (secondary) stroke events (acute } \\
\text { period) }\end{array}$ & - & & -0.150 & 0.039 & $\begin{array}{l}\text { Luengo-Fernandez et al } \\
(2013 \mathrm{a})^{19}\end{array}$ \\
\hline \multicolumn{6}{|l|}{ Other events } \\
\hline Other ICH & 2880 & 440 & 0.700 & 0.093 & \multirow{4}{*}{$\begin{array}{l}\text { Luengo-Fernandez et al } \\
(2013 a, b) \text { and Department of } \\
\text { Health }(2017)^{19-21}\end{array}$} \\
\hline Cost and disutility of CRNM & 473 & 70 & -0.058 & 0.017 & \\
\hline Cost and disutility of Gl bleed & 856 & 70 & -0.151 & 0.040 & \\
\hline Cost and disutility of other ECH & 2118 & 117 & -0.151 & 0.040 & \\
\hline Infection (related to ICM) & 757 & 297 & - & & Department of Health $(2017)^{21}$ \\
\hline \multicolumn{6}{|l|}{ Health states before any event } \\
\hline Starting utility and No-AF & - & & 0.820 & 0.008 & Data on file, Medtronic 2018 \\
\hline Disutility for presence of AF & - & & -0.014 & 0.019 & $\begin{array}{l}\text { Luengo-Fernandez et al } \\
(2013 a)^{19}\end{array}$ \\
\hline \multicolumn{6}{|l|}{ Post-stroke health states (per cycle) } \\
\hline Postmild stroke (IS or HS) & 594 & 452 & 0.727 & 0.012 & \multirow{3}{*}{$\begin{array}{l}\text { Luengo-Fernandez et al } \\
(2013 \mathrm{a}, \mathrm{b})^{1920} \ddagger\end{array}$} \\
\hline Post-moderate stroke (IS or HS) & 1158 & 1007 & 0.582 & 0.035 & \\
\hline Post-severe stroke (IS or HS) & 1759 & & 0.397 & 0.065 & \\
\hline Disutility for recurrent (secondary) stroke (post-acute period) & - & & -0.068 & 0.024 & $\begin{array}{l}\text { Luengo-Fernandez et al } \\
(2013 \mathrm{a}, \mathrm{b})^{1920}\end{array}$ \\
\hline \multicolumn{6}{|l|}{ One-time intervention costs } \\
\hline ICM acquisition and insertion & 1426 & 1129 & - & & Department of Health $(2017)^{21}$ \\
\hline ICM removal & 757 & 297 & - & & Department of Health $(2017)^{21}$ \\
\hline \multicolumn{6}{|l|}{ Monitoring and follow-up (per cycle) } \\
\hline ICM & 26.75 & & - & & Department of Health $(2017)^{21}$ \\
\hline 24 hours Holter monitoring & 37.88 & & - & & $\begin{array}{l}\text { Department of Health } \\
(2013)^{32} \dagger\end{array}$ \\
\hline \multicolumn{6}{|l|}{ Drug costs (per cycle) } \\
\hline Aspirin & 6.02 & & - & & MIMS $(2018)^{33}$ \\
\hline Warfarin & 5.71 & & - & & MIMS $(2018)^{33}$ \\
\hline Warfarin INR monitoring & 66.60 & & - & & $\begin{array}{l}\text { Dorian et al }(2014)^{14} \text { and Lip } \\
\text { et al }(2014)^{15} \S\end{array}$ \\
\hline NOAC* & 159.43 & & - & & MIMS $(2018)^{33}$ \\
\hline
\end{tabular}

*NOAC drug cost was assumed to be the average of dabigatran, rivaroxaban, apixaban, and edoxaban †Unit costs were inflated from 2013 to $2016 / 2017$ values.

fUnit costs were inflated from $2008 / 2009$ to $2016 / 2017$ values.

§Unit costs were inflated from 2014 to 2016/2017.

$\mathrm{AF}$, atrial fibrillation; $\mathrm{ECH}$, extracranial haemorrhage; GI, gastrointestinal; HS, haemorrhagic stroke; ICH, intracranial haemorrhage; ICM, insertable cardiac monitor; INR, international normalised ratio;IS, ischaemic stroke; NOAC, new oral anticoagulant; SoC, standard of care. 
Arrhythmias and sudden death

\begin{tabular}{|c|c|c|c|}
\hline & SoC & ICM & Difference \\
\hline \multicolumn{4}{|l|}{ Deterministic analysis } \\
\hline Total costs & $£ 11936$ & $£ 13360$ & $£ 1424$ \\
\hline Total IS per 100 patients & 42.38 & 37.55 & -4.83 \\
\hline QALYs & 6.304 & 6.503 & 0.199 \\
\hline Life years & 8.825 & 9.074 & 0.249 \\
\hline \multicolumn{4}{|c|}{ Probabilistic sensitivity analysis (average 95\% Crl) } \\
\hline Total costs & $\begin{array}{l}£ 12229 \\
\text { (£9720 to £15 087) }\end{array}$ & $\begin{array}{l}£ 14415 \\
\text { (£10 } 945 \text { to } £ 20350)\end{array}$ & $\begin{array}{l}£ 2186 \\
\text { (£1225 to £5263) }\end{array}$ \\
\hline Total IS per 100 patients & 41.71 (32.11 to 48.75$)$ & 37.43 (31.46 to 43.73$)$ & $-4.29(-5.03$ to -0.65$)$ \\
\hline QALYs & 6.314 (5.957 to 6.718$)$ & $6.491(6.172$ to 6.814$)$ & $0.177(0.095$ to 0.215$)$ \\
\hline Life years & $8.852(8.422$ to 9.330$)$ & 9.073 (8.718 to 9.407$)$ & $0.221(0.077$ to 0.295$)$ \\
\hline
\end{tabular}

Crl, credible interval; ICM, insertable cardiac monitor; IS, ischaemic stroke; QALYs, quality-adjusted life years; SoC, standard of care.

having a notable effect on the ICER (changes of greater than $£ 5000 /$ QALY in its value) included: only registering $\mathrm{AF}$ diagnosis for episodes lasting 5.5 hours or more; shortening the model time horizon to 10 years; shortening the model time horizon to 3 years (exceeded $£ 30$ 000/QALY in this last scenario).
A two-way sensitivity analysis simultaneously decreasing the HR of AF detection between the ICM and SoC arms and the assumed cost of monitoring for the SoC arm showed that even when assuming a HR value that is $10 \%$ of the base case (HR=3.39) and no diagnostic cost for the SoC arm, Reveal ${ }^{\circledR}$ is still cost-effective, with an ICER value

Table 5 Sub-group analysis by $\mathrm{CHADS}_{2}$ score and scenario analyses

\begin{tabular}{|c|c|c|c|c|c|}
\hline \multirow[b]{2}{*}{ Scenario description } & \multicolumn{2}{|c|}{ Total Costs } & \multicolumn{2}{|c|}{ Total QALYs } & \multirow{2}{*}{$\begin{array}{l}\text { ICER } \\
\text { ICM versus } \\
\text { SoC }\end{array}$} \\
\hline & SoC & ICM & SoC & ICM & \\
\hline Base-case analysis & $£ 11936$ & $£ 13360$ & 6.304 & 6.503 & $£ 7140$ \\
\hline \multicolumn{6}{|l|}{ Subgroup analyses* } \\
\hline $\mathrm{CHADS}_{2}$ score 2 & $£ 10654$ & $£ 12332$ & 7.508 & 7.665 & $£ 10735$ \\
\hline $\mathrm{CHADS}_{2}$ score 3 & $£ 12130$ & $£ 13591$ & 5.960 & 6.133 & $£ 8425$ \\
\hline $\mathrm{CHADS}_{2}$ score 4,5 and 6 & $£ 11629$ & $£ 13070$ & 5.375 & 5.527 & $£ 9463$ \\
\hline \multicolumn{6}{|l|}{ Scenario analyses } \\
\hline Choice of $O A C=$ warfarin & $£ 11896$ & $£ 12971$ & 6.298 & 6.434 & $£ 7900$ \\
\hline Treatment discontinuation for reasons other than bleeding $=0 \%$ & $£ 11959$ & $£ 13570$ & 6.316 & 6.680 & $£ 4427$ \\
\hline HR ICM vs SoC (diagnostic yield) $=8.78^{34}$ & $£ 11836$ & $£ 13330$ & 6.348 & 6.518 & $£ 8793$ \\
\hline $\begin{array}{l}\text { Monitoring costs for } \mathrm{SoC}=\text { assume pulse check and HR of ICM versus } \\
\mathrm{SoC}=1 / 24 \text { th of the Holter monitoring (scenario proposed by clinical experts) }\end{array}$ & $£ 10639$ & $£ 12808$ & 6.287 & 6.497 & $£ 10323$ \\
\hline $\begin{array}{l}\text { Monitoring costs for } \mathrm{SoC}=\text { assume pulse check and base-case HR of ICM vs } \\
\mathrm{SoC}=33.9\end{array}$ & $£ 10643$ & $£ 12811$ & 6.304 & 6.503 & $£ 10874$ \\
\hline AF episode duration lasting for $\geq 5.5$ hours $\dagger$ & $£ 10015$ & $£ 11737$ & 6.644 & 6.741 & $£ 17693$ \\
\hline OAC uptake after AF diagnosis $=66.35 \%{ }^{510}$ & $£ 11923$ & $£ 13516$ & 6.302 & 6.445 & $£ 11145$ \\
\hline Time horizon $=3$ years & $£ 2257$ & $£ 4142$ & 2.219 & 2.232 & $£ 139742$ \\
\hline Time horizon $=5$ years & $£ 3966$ & $£ 5669$ & 3.294 & 3.331 & $£ 45916$ \\
\hline Time horizon $=10$ years & $£ 7969$ & $£ 9363$ & 5.136 & 5.247 & $£ 12512$ \\
\hline Time horizon $=25$ years & $£ 11923$ & $£ 13345$ & 6.301 & 6.500 & $£ 7146$ \\
\hline
\end{tabular}

*Each $\mathrm{CHADS}_{2}$ subgroup will differ on ischaemic stroke risk, diagnostic accuracy of monitoring strategies, as well as the corresponding age and gender mix of the group in the REVEAL AF trial.

†Analyses using the alternative definition of AF episode were carried out using the REVEAL AF clinical data set (data on file, Medtronic 2018).

AF, atrial fibrillation; ICER, incremental cost-effectiveness ratio; ICM, insertable cardiac monitor; OAC, oral anticoagulation; QALY, quality-adjusted life-year; SoC, standard of care. 


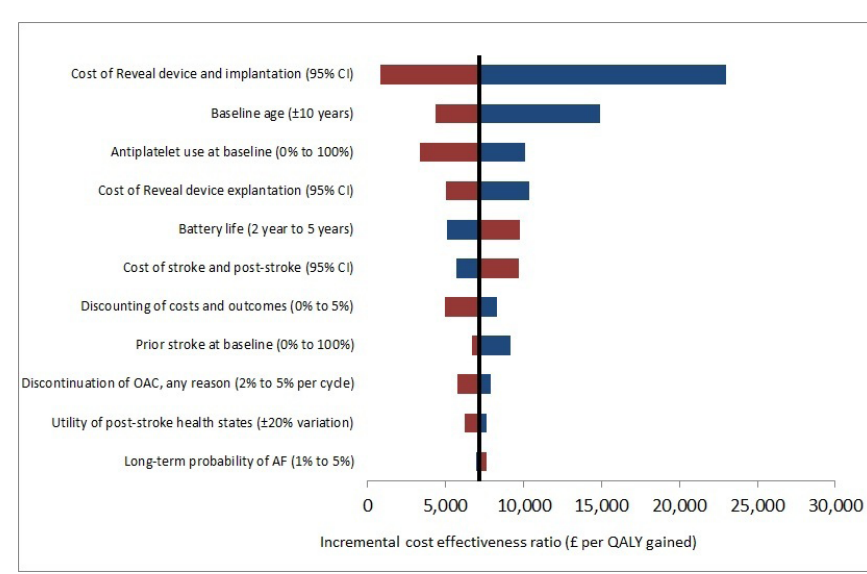

Figure 3 Tornado diagram. Note: red bars reflect the incremental cost-effectiveness ratio impact of the low limit of the parameter intervals considered, while blue bars reflect the impact of the high limit value of the parameter intervals.

of 118574 per QALY gained (see online supplementary file 8$)$.

\section{Sensitivity analysis}

Model sensitivity was tested by varying each parameter and recording the resulting ICERs. The results of these analyses are summarised in the tornado diagram in figure 3, which shows the top 10 most sensitive parameters. One of the most sensitive parameters was, as expected, the cost of the Reveal ${ }^{\circledR}$ device and implantation procedure, which increased the ICER to over $£ 20000 /$ QALY when using the upper limit of the parameter. The model is also sensitive to baseline population characteristics, such as age, antiplatelet use and prior stroke history, although to a lesser degree. Other cost assumptions, such as cost of ICM device explantation, or cost of stroke and post-stroke health states, had a significant impact on the model results. The remaining scenarios had a smaller impact on the ICER $( \pm £ 2000 /$ QALY variation around the base-case value), maintaining results well within the willingness to pay threshold.

\section{DISCUSSION}

\section{Overview}

Our analyses found that continuous cardiac monitoring using ICM in a high-risk population at risk of, but without $\mathrm{AF}$ was a cost-effective use of resources in the UK NHS setting, with the base-case ICER and all but one of the sensitivity analysis ICERs falling below the threshold used by NICE. The ICER for ICM in this model appeared lower than ICERs estimated for ICM in patients with cryptogenic stroke ${ }^{6}$ or unexplained syncope. ${ }^{23}$

\section{Sensitivity一duration of AF}

The base-case model assumed that detecting an episode of $\mathrm{AF}$ at least $6 \mathrm{~min}$ in duration would warrant the commencement of anticoagulation therapy. The frequency and duration of AF can differ from patient to patient, ${ }^{24}$ and if we accept the stasis-based causal mechanism between $\mathrm{AF}$ and stroke, ${ }^{2}$ then there is merit in discussing how much AF, and how often, is enough to warrant anticoagulation.

Although multiple factors are known to be predictive of stroke in addition to the duration and frequency of $\mathrm{AF}^{25}$ our model assumption for a 6 min AF duration threshold is generally supported by findings from other trials and UK expert opinion, ${ }^{26-30}$ if one takes into account differences in the patient risk profiles, clinical histories and management strategies in those trials.

One of our scenario analyses assumed that only patients with AF lasting longer than 5.5 hours (a figure based on the TRENDS study ${ }^{26}$ had increased stroke risk and received anticoagulation; this scenario produced an ICER which was higher than base case but still well below the NICE threshold (table 5).

\section{Anticoagulation therapy following AF diagnosis}

The cost-effectiveness of a diagnostic strategy depends not only on its accuracy and cost but also the costs and benefits of timely and accurate diagnosis, which in this case derived from receipt of appropriate treatment. Two scenario analyses illustrated how the cost-effectiveness of ICM monitoring is sensitive to assumptions around the proportion who take up and the proportion who persist with appropriate treatment following $\mathrm{AF}$ detection. The base-case model reflects the NICE Clinical Guideline for Management of AF (CG180) ${ }^{9}$ in assuming all patients at high risk of stroke in whom $\mathrm{AF}$ is detected will start on OAC. However, in REVEAL AF, and the comparable study PREDATE AF, ${ }^{10}$ one-third of patients remained on only antiplatelet therapy even after AF diagnosis. Under this assumption, the ICER increased to £11 145/QALY but remained well below the cost-effectiveness threshold. The base case also assumed that $16 \%$ of patients, beyond those who discontinued due to major bleeding, would stop treatment each year. If adherence is improved, ICM monitoring becomes even more cost-effective.

\section{Standard monitoring - use of Holter monitors}

Our model assumed the current SoC to be a single 24 hours Holter monitor administered annually. By contrast, the NICE Clinical Guideline for AF management recommends only an opportunistic pulse check for these at-risk patients. However, current clinical practice for high-risk patients is likely to fall somewhere between opportunistic pulse checks and an annual 24 hours Holter. Our scenario analyses show that ICM is likely to represent better value to the NHS than either strategy.

\section{CONCLUSIONS}

This analysis shows that the use of an ICM is likely to be a cost-effective means of detecting incident $\mathrm{AF}$ and identifying candidates for oral anticoagulation, with the aim of preventing IS. This cost-effectiveness would apply in the UK setting as well as other healthcare systems that fund interventions based on clinical evidence and comparative cost-effectiveness. 
Currently, ongoing research may provide clearer inputs for some of our modelling assumptions, but based on the sensitivity analyses we have conducted, the overall conclusion with respect to cost-effectiveness is unlikely to change.

Acknowledgements The authors would like to thank Jana Tillotson for editorial support.

Contributors CR, LS, AD, MQ and ST conceived the analysis. CR and LS conducted the analysis. PZ, ME, MR and KW validated the model structure, assumptions and inputs. CR and LS wrote the introduction, methods and results. ST, CR and LS wrote the discussion section. All authors provided critical feedback on the manuscript and have approved the final version.

Funding REVEAL AF and this cost-effectiveness analysis were funded by Medtronic plc.

Competing interests CR, LS and AD are employed by Symmetron Ltd, which received funding from Medtronic plc for this analysis. ME's institution has received payments from Medtronic plc for ME's participation in this analysis. KW and MR have received consultancy fees from Medtronic plc. ST, PZ and CW are employees and have equity interest in Medtronic plc. MQ was a Medtronic employee at the time of the analysis.

Patient consent for publication Not required.

Provenance and peer review Not commissioned; externally peer reviewed.

Data availability statement All data relevant to the study are included in the article or uploaded as supplementary information.

Open access This is an open access article distributed in accordance with the Creative Commons Attribution Non Commercial (CC BY-NC 4.0) license, which permits others to distribute, remix, adapt, build upon this work non-commercially, and license their derivative works on different terms, provided the original work is properly cited, appropriate credit is given, any changes made indicated, and the use is non-commercial. See: $\mathrm{http}: / /$ creativecommons.org/licenses/by-nc/4.0/.

\section{REFERENCES}

1. Wolf PA, Abbott RD, Kannel WB. Atrial fibrillation as an independent risk factor for stroke: the Framingham study. Stroke 1991;22:983-8.

2. Kamel H, Okin PM, Elkind MSV, et al. Atrial fibrillation and mechanisms of stroke: time for a new model. Stroke; a journal of cerebral circulation 2016;47:895-900.

3. Strickberger SA, Ip J, Saksena S, et al. Relationship between atrial tachyarrhythmias and symptoms. Heart Rhythm 2005;2:125-31.

4. Arya A, Piorkowski C, Sommer P, et al. Clinical implications of various follow up strategies after catheter ablation of atrial fibrillation. Pacing Clin Electrophysiol 2007;30:458-62.

5. Reiffel JA, Verma A, Kowey PR, et al. Incidence of previously undiagnosed atrial fibrillation using Insertable cardiac monitors in a high-risk population. JAMA Cardiology 2017;2:1120-7.

6. Diamantopoulos A, Sawyer LM, Lip GYH, et al. Cost-effectiveness of an insertable cardiac monitor to detect atrial fibrillation in patients with cryptogenic stroke. International Journal of Stroke 2016;11:302-12.

7. National Institute for Health and Care Excellence. Guide to the methods of technology appraisal. London: National Institute for Health and Care Excellence, 2013.

8. Hindricks G, Pokushalov E, Urban L, et al. Performance of a new Leadless implantable cardiac monitor in detecting and quantifying atrial fibrillation results of the Xpect trial. Circ Arrhythm Electrophysiol 2010;3:141-7.

9. National Institute for Health and Care Excellence. Atrial fibrillation: management. Clinical guideline [CG180], 2014.

10. Nasir JM, Pomeroy W, Marler A, et al. Predicting determinants of atrial fibrillation or flutter for therapy elucidation in patients at risk for thromboembolic events (PREDATE AF) study. Heart Rhythm 2017;14:955-61.
11. Tawfik A, Bielecki J, Krahn M, et al. Systematic review and network meta-analysis of stroke prevention treatments in patients with atrial fibrillation. Clinical pharmacology : advances and applications 2016;8:93-107.

12. Gage BF, van Walraven $C$, Pearce $L$, et al. Selecting patients with atrial fibrillation for anticoagulation: stroke risk stratification in patients taking aspirin. Circulation 2004;110:2287-92.

13. Pisters R, Lane DA, Marin F, et al. Stroke and thromboembolism in atrial fibrillation. Circulation Journal 2012;76:2289-304.

14. Dorian $\mathrm{P}$, Kongnakorn $\mathrm{T}$, Phatak $\mathrm{H}$, et al. Cost-effectiveness of apixaban vs. current standard of care for stroke prevention in patients with atrial fibrillation. European Heart Journal 2014;35:1897-906.

15. Lip GYH, Kongnakorn T, Phatak $\mathrm{H}$, et al. Cost-effectiveness of apixaban versus other new oral anticoagulants for stroke prevention in atrial fibrillation. Clinical Therapeutics 2014;36:192-210.

16. Claassen DO, Kazemi N, Zubkov AY, et al. Restarting anticoagulation therapy after warfarin-associated intracerebral hemorrhage. Archives of Neurology 2008;65:1313-8.

17. Sorensen SV, Dewilde S, Singer DE, et al. Cost-effectiveness of warfarin: Trial versus "real-world" stroke prevention in atrial fibrillation. American Heart Journal 2009;157:1064-73.

18. Office for National Statistics. Mortality statistics: deaths: underlying cause, sex and age-group, 2014: chapter IX. Diseases of the circulatory system. England and Wales, 2014. Available: http://www. ons.gov.uk/ons/rel/vsob1/mortality-statistics-deaths-registered-inengland-and-wales-series-dr-/2012/index.html

19. Luengo-Fernandez R, Gray AM, Bull L, et al. Quality of life after TIA and stroke: ten-year results of the Oxford Vascular Study. Neurology 2013;81:1588-95.

20. Luengo-Fernandez R, Paul NL, Gray AM, et al. Population-based study of disability and institutionalization after transient ischemic attack and stroke: 10-year results of the Oxford Vascular Study. Stroke; a journal of cerebral circulation 2013;44:2854-61.

21. Department of Health. NHS reference costs 2016-2017, 2017. Available: https://improvement.nhs.uk/resources/reference-costs/

22. Reiffel JA, Verma A, Kowey P, et al. A comparison of atrial fibrillation monitoring strategies in patients at high risk for atrial fibrillation and stroke: results from the reveal AF study. J Am Coll Cardiol 2018;71.

23. Davis S, Westby M, Pitcher D, et al. Implantable loop recorders are cost-effective when used to investigate transient loss of consciousness which is either suspected to be arrhythmic or remains unexplained. Europace 2012;14:402-9.

24. Martinek M, Aichinger J, Nesser H-J, et al. New insights into long-term follow-up of atrial fibrillation ablation: full disclosure by an implantable pacemaker device. J Cardiovasc Electrophysiol 2007:18:818-23.

25. Botto GL, Padeletti L, Santini M, et al. Presence and duration of atrial fibrillation detected by continuous monitoring: crucial implications for the risk of thromboembolic events. J Cardiovasc Electrophysiol 2009;20:241-8

26. Glotzer TV, Daoud EG, Wyse DG, et al. The relationship between daily atrial tachyarrhythmia burden from implantable device diagnostics and stroke risk. Circ Arrhythm Electrophysiol 2009;2:474-80.

27. Healey JS, Connolly SJ, Gold MR, et al. Subclinical atrial fibrillation and the risk of stroke. N Engl J Med 2012;366:120-9.

28. National Institute for Health and Care Excellence. Reveal LINQ insertable cardiac monitor to detect atrial fibrillation after cryptogenic stroke. Medtech innovation briefing [MIB141]. London: National Institute for Health and Care Excellence, 2018.

29. Healey JS, Alings M, Ha A, et al. Subclinical atrial fibrillation in older patients. Circulation 2017:136:1276-83.

30. Wong JA, Conen D, Van Gelder IC, et al. Progression of DeviceDetected Subclinical Atrial Fibrillation and the Risk of Heart Failure. $J$ Am Coll Cardiol 2018;71:2603-11.

31. Ariesen MJ, Claus SP, Rinkel GJE, et al. Risk factors for intracerebral hemorrhage in the general population. Stroke 2003;34:2060-5.

32. Department of Health. NHS reference costs 2012-2013, 2013. Available: https://www.gov.uk/government/publications/nhsreference-costs-2012-to-2013

33. Monthly Index of Medical Specialities (MIMS). Online version, 2018. Available: http://www.mims.co.uk/

34. Sanna T, Diener H-C, Passman RS, et al. Cryptogenic stroke and underlying atrial fibrillation. N Engl J Med 2014;370:2478-86. 